CARDIOVASCULAR MEDICINE

\title{
Management of chronic heart failure in the community: role of a hospital based open access heart failure service
}

\author{
S Shah, M K Davies, D Cartwright, P Nightingale
}

Heart 2004;90:755-759. doi: 10.1136/hrt.2002.006460

See end of article for authors' affiliations

(1)

Correspondence to:

Dr Saeed Shah, 17 South

Street, Harborne,

Birmingham B17 ODB, UK; saeedshah@miranshah.

freeserve.co.uk

Accepted 3 October 2003

\begin{abstract}
Objective: To evaluate the role of an open access heart failure service based at a teaching hospital for the diagnosis and treatment optimisation of patients with heart failure in the community and to identify measures that may further enhance the effectiveness of such a service.

Subjects: 963 patients with suspected heart failure seen over an eight year period referred by their general practitioners to the cardiology department at a district general hospital.

Main outcome measures: Presence or absence of left ventricular systolic dysfunction (LVSD) (left ventricular ejection fraction $<50 \%$ on echocardiography), and determination of the risk factors and predictors of LVSD.

Results: The majority of the patients were women $(60 \%$ v $40 \%)$ and elderly (mean age 68.8 years). On echocardiography, only $30.8 \%$ were found to have LVSD. Patients were more likely to have LVSD if they were men $(42.3 \% \vee 23.1 \%, p<0.001$, relative risk (RR) 1.8$)$, were $>60$ years of age $(33.5 \% \vee 20.8 \%$, $p<0.001$, RR 1.6), or had a history of diabetes $(49.4 \% v 29.1 \%, p<0.001$, RR 1.7), ischaemic heart disease $(36.5 \%$ v $29.1 \%, p=0.04$, RR 1.3), or atrial fibrillation $(52.6 \% v 27.8 \%, p<0.001$, RR 1.9). An abnormal ECG $(48.4 \%$ v 19.5\%, $\mathrm{p}<0.001$, RR 2.5$)$ and cardiothoracic ratio $>0.5$ on chest radiograph $(44.3 \%$ v $17.8 \%, p<0.001$, RR 2.5) were found to be good predictors of LVSD. A normal ECG (negative predictive value $80.5 \%$ ) and a cardiothoracic ratio of $<0.5$ (negative predictive value $82.2 \%$ ) can be used as baseline measures to identify patients with lower risk of developing LVSD (combined negative predictive value $87.9 \%)$.

Conclusions: An open access heart failure clinic is effective for the diagnosis and management of chronic heart failure in community based patients. The presence of risk factors and simple baseline tests can be used to identify patients with LVSD in the community. The introduction of a protocol based on these findings into a referral system can improve the efficiency and cost effectiveness of such a service.
\end{abstract}

$\mathrm{H}$ eart failure is a common and disabling condition with a considerable economic burden. ${ }^{12}$ Estimates of the prevalence of symptomatic heart failure in the general European population range from $0.4-2 \%{ }^{3}$ The most common cause of chronic heart failure is left ventricular systolic dysfunction (LVSD). ${ }^{4}$ About 3\% of the adult population have systolic dysfunction but half of them are asymptomatic. ${ }^{5}$ The prognosis of symptomatic LVSD is poor $(30 \%$ mortality within one year, increasing to $60-70 \%$ at five years). ${ }^{6}$ Recent studies have also confirmed poor long term prognosis even for patients with asymptomatic LVSD. ${ }^{6}$

Identification and treatment of patients with LVSD improves survival and reduces morbidity. ${ }^{78}$ The clinical diagnosis of LVSD, however, is fraught with difficulties. ${ }^{9}$ There is evidence that a significant number of patients with heart failure in the UK are not being treated appropriately. ${ }^{10}$ Of these, a substantial number do not receive optimal treatment. ${ }^{11}$ On the other hand, it has also been shown that for a significant number of patients heart failure is wrongly diagnosed and treated when they have noncardiac diseases. ${ }^{12}$ Although diagnosis of obvious signs and symptoms is relatively easy, several studies have shown that the accuracy of diagnosis by clinical means alone is often inadequate. This is particularly the case for women, the elderly, patients with obstructive airways disease, and the obese. $^{13}$

Transthoracic echocardiography is considered to be one of the most sensitive, specific, and easily performed and reproducible tools for the diagnosis of LVSD. ${ }^{14}$ This facility, however, is not generally available for a large number of potential patients with heart failure in the community. Hence, large numbers of patients suspected to have heart failure never get this diagnosis verified. Open access echocardiography services have been established in several British and European centres for this purpose. ${ }^{15}$ Their data showed a significant benefit of such service to the community. Overall though, the numbers of patients being screened by these services are small. Many, in addition, are technician run services and thus the correct interpretation and advice on the future management of patients are not always available.

Considering these aspects, a cardiologist led open access heart failure service was established in 1994 at the cardiology department in a local teaching hospital. The main objective is to provide to the local general practitioners a rapid, one stop service for diagnosis and advice on further management of patients suspected to have heart failure in the community. So far more than 1200 patients have been seen in this clinic. It is one of the biggest and longest running services of its kind in the UK. All patients referred by the general practitioners are seen and evaluated by a physician (consultant cardiologist and specialist registrar/ research fellow). The evaluation is followed by a 12 lead ECG, a chest radiograph, and echocardiographic examination. An individualised management plan is thereafter advised and a letter is sent to the referring doctor within a week. If indicated, patients are followed up in the general or specialist heart failure clinics for further management and treatment optimisation. Here we evaluate the service and suggest methods for streamlining the referral of patients to the clinic. 


\section{METHODS}

\section{Patient cohort}

Our study cohort consisted of patients referred by their general practitioners to the open access heart failure clinic between 1994 and 2001. Forty three local general practitioners were invited to refer patients with symptoms and signs suggestive of heart failure (dyspnoea, fatigue, oedema); patients who were already managed for having heart failure but never had the diagnosis of heart failure confirmed previously; and patients with factors associated with increased risk of developing heart failure or asymptomatic LVSD (previous myocardial infarction, coronary artery bypass grafting, or longstanding hypertension with ECG evidence of left ventricular hypertrophy, cardiac murmurs, and atrial fibrillation).

\section{Service setup}

The clinic was based at the cardiology department of the local teaching hospital. The staff consisted of a cardiac technician, a middle grade cardiologist (specialist registrar, research registrar), and the consultant cardiologist. Three to four patients were seen in a session with just one session per week. Later, a weekly treatment optimisation clinic was also set up where patients who required initiation and adjustment of heart failure treatment were seen and followed up. This clinic was run by the heart failure specialist nurse and a middle grade cardiologist. On average eight patients were seen in this clinic, which included new and follow up cases.

\section{Questionnaire/history}

A detailed history was taken. All patients were asked about their symptoms, exercise tolerance, relevant illnesses (ischaemic heart disease, hypertension, valvar disease, diabetes, asthma and chronic obstructive airways disease, history of rheumatic fever, palpitations, and gastro-oesophageal reflux/ hiatus hernia), medications, smoking, and alcohol consumption. A patient's functional class was determined by the New York Heart Association classification.

\section{Physical examination}

A full physical examination was performed including measurement of resting pulse and blood pressure.

\section{ECG}

All patients had a 12 lead ECG recorded .Abnormalities such as Q wave, ST segment, and T wave abnormalities, left ventricular hypertrophy, atrial hypertrophy, bundle branch block or other conduction abnormalities, and atrial fibrillation/flutter or other rhythm abnormalities were recorded. All of these caused an ECG to be classified as abnormal.

\section{Chest radiography}

All patients had posteroanterior view chest radiography. The cardiothoracic ratio was recorded. To obtain accurate measurement, a vertical line was drawn on the radiograph through the midpoint of the spine from the sternum to the diaphragm. The maximum transverse diameter of the heart was obtained by adding the widest distance of the right heart border from the midline and the left heart border to the midline. This value was then divided by the maximum transverse diameter of the thorax.

\section{Echocardiogram}

All patients had transthoracic echocardiography performed by a technician, specialist registrar, or research fellow and in all cases reviewed by the consultant cardiologist (MKD). If required, the study was extended or repeated by the consultant. Standard two dimensional, colour, and Doppler echocardiograms (Toshiba/Hewlett Packard) were recorded with patients recumbent at about $40^{\circ}$. M mode measurements
Table 1 Baseline characteristics of study population

\begin{tabular}{llll}
\hline & Men & Women & $\begin{array}{l}\text { p Value (men v } \\
\text { women) }\end{array}$ \\
\hline Total & $386(40.1 \%)$ & $577(59.9 \%)$ & \\
Mean (SD) age (years) & $68.5(12.1)$ & $69.0(12.8)$ & 0.52 \\
IHD & $22 \%$ & $24 \%$ & 0.45 \\
Hypertension & $39 \%$ & $46 \%$ & 0.06 \\
Diabetes & $9 \%$ & $7 \%$ & 0.30 \\
Atrial fibrillation & $18 \%$ & $8 \%$ & $<0.001$ \\
Asthma & $7 \%$ & $12 \%$ & 0.01 \\
COAD & $14 \%$ & $7 \%$ & 0.001 \\
\hline
\end{tabular}

$C O A D$, chronic obstructive airways disease; IHD, ischaemic heart disease.

were made from the parasternal long axis view. Left ventricular ejection fraction was estimated indirectly from the fractional shortening; in some cases the biplane disc summation method (Simpson's rule) and regional wall motion index were used. For patients with suboptimal views, eyeball estimation of ejection fraction and left ventricular systolic function was used. Although some characteristics of diastolic dysfunction were determined, systolic function was the main diagnostic module used. An ejection fraction of 50\% or more was used to define good systolic function.

\section{Statistical analysis}

All statistical analysis was done with SPSS for Windows (version 10, SPSS Inc, Chicago, Illinois, USA). Differences between groups were explored by unpaired $t$ tests for continuous data and $\chi^{2}$ for categorical data. Pearson's correlation coefficient was used to quantify relations between continuous variables and Kendall's tau $\mathrm{b}_{\mathrm{b}}$ was used for relations between continuous and ordinal variables. Predictive markers of LVSD were identified by stepwise logistic regression analysis.

\section{RESULTS}

All invited general practitioners used this service. So far, more than 1200 patients have been seen in the clinic. We analysed the records of the first 963 patients seen in this service between 1994 and 2001. Table 1 shows the baseline characteristics of the patient cohort. Patients, although elderly (average age 68.8 years), were relatively younger than similar cohorts with a variety of co-morbid conditions. Hypertension was the most common back ground condition in both sexes (men 39\%, women $46 \%$ ). It was followed by the presence of ischaemic heart disease (previous myocardial infarction or myocardial revascularisation procedure, angina) in over $20 \%$, with a similar male to female proportion. Respiratory conditions were also common. Asthma and chronic obstructive airways disease was present in almost a fifth of this population. A higher proportion of men had chronic obstructive airways disease ( $14 \% v 7 \%, \mathrm{p}<0.001)$.

Table 2 Probable diagnoses among patients with preserved left ventricular systolic function

\begin{tabular}{lc}
\hline Condition & Percentage of patients \\
\hline Hypertensive heart disease & 19.2 \\
Obstructive airways diseases* & 11 \\
Atrial fibrillation & 6.3 \\
Valvar heart disease & 5.4 \\
Ischaemic heart disease & 3.3 \\
Significant others† & 2.3 \\
Possible others & 2.7 \\
No obvious cause established & 49.5 \\
\hline
\end{tabular}

*COAD and asthma; †pulmonary fibrosis, pleural effusion, carcinoma, obesity; łperipheral vascular disease (varicose veins or arterial insufficiency) medications, arthritis, chronic lymphoedema, etc. 


\begin{tabular}{|c|c|c|c|}
\hline $\begin{array}{ll}\text { Table } 3 & \text { Predictors c } \\
\text { (LVSD) } & \end{array}$ & fleft ventric & Jlar systc & olic dysfunction \\
\hline Factors & $\begin{array}{l}\text { Percentage } \\
\text { with LVSD }\end{array}$ & $\begin{array}{l}\text { Relative } \\
\text { risk }\end{array}$ & $\begin{array}{l}\text { p Value } \\
\text { (condition } \\
\text { present } v \text { absent) }\end{array}$ \\
\hline Men $v$ women & $42.3 \vee 23.1$ & 1.8 & $<0.001$ \\
\hline Age $>60 v<60$ years & $33.5 \vee 20.8$ & 1.6 & $<0.001$ \\
\hline$A F \vee n o A F$ & $52.6 \vee 27.8$ & 1.9 & $<0.001$ \\
\hline Diabetes $v$ no diabetes & $49.4 \vee 29.1$ & 1.7 & $<0.001$ \\
\hline IHD v no IHD & $36.5 \vee 29.1$ & 1.3 & 0.04 \\
\hline $\mathrm{CTR}>0.5 v<0.5$ & $44.3 \vee 17.8$ & 2.5 & $<0.001$ \\
\hline Abnormal $v$ normal ECG & $48.4 \times 19.5$ & 2.5 & $<0.001$ \\
\hline
\end{tabular}

Only $30.8 \%$ of the patients were found to have LVSD (left ventricular ejection fraction less than $50 \%$ ). In patients with normal systolic function and symptoms suggestive of heart failure (table 2), 19.2\% were diagnosed to have hypertensive heart disease (history of longstanding hypertension with the presence of left ventricular hypertrophy, normal left ventricular systolic function, abnormalities of diastolic filling, and various degrees of valvar abnormalities on echocardiography), 11\% obstructive airways diseases (asthma and chronic obstructive airways disease), 6.3\% atrial fibrillation, 5.4\% symptomatic valvar disease, and $3.3 \%$ ischaemic heart disease (angina equivalent). Various other diagnoses $(2.3 \%)$ were pulmonary fibrosis, lung neoplasm, pleural effusion, and probable cardiac amyloidosis. In a small number of patients, obesity, arthritis, peripheral vascular disease (arter$\mathrm{ial} /$ venous), and use of certain medications (non-steroidal anti-inflammatory drugs, calcium channel antagonists, oral steroids, etc) were considered to be the cause of symptoms, particularly of peripheral oedema. In almost half of this population with normal left ventricular systolic function, no apparent cause could be identified.

Dyspnoea, which was the main symptom the patients were referred with, was found to be a poor predictor of LVSD. LVSD was more common in men than in women $(42.3 \% \mathrm{v}$ $23.1 \%, \mathrm{p}<0.001)$ and in patients older than 60 years than in patients younger than 60 years $(33.5 \% v 20.8 \%$, p $<0.001)$ (table 3). LVSD was more common if there was an abnormal ECG than if the ECG was normal (48.4\% $v 19.5 \%, \mathrm{p}<0.001)$ and among patients with atrial fibrillation $(52.6 \% v 27.8 \%$, $\mathrm{p}<0.001)$. A high cardiothoracic ratio on the chest radiograph was also found to be an important predictor of cardiac dysfunction $(44.3 \% \vee 17.8 \%, p<0.001)$. Left ventricular systolic impairment was also more common in those with diabetes than in those without $(49.4 \% \vee 29.1 \%$, p < 0.001$)$ and in patients with established ischaemic heart disease (36.5\% $v 29.1 \%, \mathrm{p}=0.04)$.

A large number of patients were taking diuretics and other cardiac medications at the time of their referral (table 4). A very small proportion of these patients were taking angiotensin converting enzyme (ACE) inhibitors in the early phase of the service (1994-1997) with substantial increase in the later phase. Similarly, $\beta$ blockers were used by a very small number of patients and mainly for treating hypertension and angina. There was some increase in their use in the later phase of the clinic, but it was mainly for ischaemic heart disease. Lipid lowering agents were not included initially; however, their use increased manifold in the last few years of the study period. Significant changes were made in patients' medications (table 4) and their overall management.

Stepwise logistic regression analysis identified four significant factors associated with LVSD. They were abnormal ECG, cardiomegaly on the chest radiograph (cardiothoracic ratio greater than 0.5 ), male sex, and history of diabetes .The logistic regression model predicted that if patients have none of these "risk factors" the probability of having LVSD is 0.08 . However, if they have exactly one risk factor, the probability is between 0.14 and 0.24 (depending on which risk factor they have); if they have two risk factors, the probability is 0.29 to 0.46 . For three risk factors, the probability is 0.53 to 0.69 , which increases to 0.80 for all four risk factors.

\section{DISCUSSION}

In line with previous studies, our work shows that the majority of patients with clinically diagnosed heart failure have normal left ventricular systolic function on echocardiography. ${ }^{15-16}$ In our cohort; only $30.8 \%$ of the referred patients were found to have impaired LVSD. In the group with symptoms but with preserved left ventricular systolic function, only half were found to have a probable cause that could have been responsible for their symptoms (table 2). No cause was found for symptoms in the other half. The reason for such a high proportion of these symptomatic patients with preserved left ventricular systolic function is probably poor patient selection on the part of referring practitioners and the general characteristics of such a cohort. Diagnosis of heart failure secondary to LVSD on the basis of usual symptoms and signs (dyspnoea, poor effort tolerance, peripheral oedema) can be difficult in the elderly, the obese, patients with chest conditions, and women. ${ }^{9}$ The majority of our patients were women $(60 \%)$ and relatively older (average age 68.8 years), and a significant proportion had chronic lung diseases $(11 \%)$. These predisposing factors may explain the high proportion of patients in our study who had symptoms

Table 4 Patient previous medications and the amendments made

\begin{tabular}{llllc}
\hline Drug & $\begin{array}{l}\text { Referred patients taking } \\
\text { medication (\%) }\end{array}$ & $\begin{array}{l}\text { Medication } \\
\text { stopped (\%) }\end{array}$ & $\begin{array}{l}\text { Medication initiated } \\
\text { and titrated (\%) }\end{array}$ & $\begin{array}{l}\text { Medication dosage } \\
\text { altered (\%) }\end{array}$ \\
\hline ACE inhibitors & 21 & $2.1^{*}$ & $16.5 \dagger$ & 7.4 \\
B Blocker & 9.9 & $0.3 \ddagger$ & $15.1 \S$ & 3.6 \\
Aspirin & 18.9 & - & 32 & 2.9 \\
Diuretics & 57.1 & 3.7 & 4.3 & $13.5^{* *}$ \\
Digoxin & 9.7 & 0.6 & 1.7 & 4.3 \\
CCA & 15.2 & 0.2 & 4.7 & 3.3 \\
Warfarin & 1.2 & - & 5.4 & - \\
Nitrates & 23.4 & 1.3 & 3.3 & 7.2 \\
Bronchodilators & 13 & - & 4.3 & - \\
\hline
\end{tabular}

Statins use was not recorded in the earlier phase of the service.

*Intolerance, significant renal impairment, side effects; tincludes patients with valvar dysfunction, hypertensive heart disease, diabetes; $¥ s i d e$ effects particularly bronchospasm or significant peripheral arterial disease; Sincludes patients with angina, heart failure, atrial fibrillation (rate control, rhythm maintenance), hypertension; - loop and thiazide diuretics, for hart failure and hypertension; ${ }^{* *}$ loop diuretic changed to thiazide or vice versa or dosage of diuretic reduced or increased.

$\mathrm{ACE}$, angiotensin converting enzyme; $\mathrm{CCA}$, calcium channel antagonists. 
(mainly dyspnoea) but were found to have normal left ventricular systolic function on the echocardiogram.

In our cohort, the majority (about $60 \%$ ) of the referred patients were women. In most of the previous studies, there was usually a higher proportion of men than of women. ${ }^{1}{ }^{14}$ However, in keeping with these studies, men were found to have a relatively higher proportion of LVSD than were women despite having similar incidences of ischaemic heart disease, diabetes, hypertension, and age (table 1). The higher proportion of LVSD in men can probably be attributed to a higher incidence of atrial fibrillation and indirectly to smoking (there was a higher incidence of chronic obstructive airways disease among men).

Similar to previous studies, in our study we found a strong relation between LVSD and co-morbidities such as presence of ischaemic heart disease, atrial fibrillation, and diabetes mellitus (table 3). ${ }^{17}$ Ischaemic heart disease has been shown to confer a fourfold increase in the risk for developing heart failure. ${ }^{18}$ It is believed to be the underlying cause in about two thirds of patients with heart failure caused by LVSD. In our cohort, however, we found ischaemic heart disease to confer relatively low but significant relative risk for LVSD. The fact that our definition of ischaemic heart disease encompassed patients with various degrees of this condition may explain this aberration. Secondly, given the significant improvement in the management of ischaemic heart disease, a substantial number of patients with a history of myocardial infarction were already taking ACE inhibitors, nitrates and other vasodilators, aspirin, and in some cases statins and $\beta$ blockers. These medications might have prevented the progression of LVSD by altering the course of left ventricular remodelling and dilatation and by preventing further ischaemia.

Atrial fibrillation, on the other hand, was one of the most important factors associated with LVSD. The prevalence of this arrhythmia increases with age and up to $10 \%$ of the population have this condition by the age of 80 years. ${ }^{1{ }^{17}}$ It is also associated with other conditions such as coronary heart disease, hypertension, cardiomyopathies, valvar heart disease, and chronic chest conditions. Hence, the increased incidence of cardiac impairment in patients with atrial fibrillation may be related to these co-morbid conditions. However, atrial fibrillation can also induce cardiac remodelling in otherwise disease-free hearts by various mechanisms leading to heart failure or LVSD. ${ }^{19}$

Diabetes was another important risk factor and in the multivariate analysis it was found to be more significant than the presence of coronary artery disease or hypertension. Just over $8 \%$ of the patient cohort had known diabetes. In other studies, diabetes was present in up to $20 \%$ of patients, with a similar number of patients having glucose intolerance. ${ }^{20}$ However, as we relied on patient history and did not screen for this metabolic abnormality, the proportion of patients with diabetes in our cohort may have been underestimated. We did not find any significant difference between the male and female populations. Diabetes was found to be an independent risk factor for developing LVSD after the correction in this subgroup for the presence of coronary artery disease, hypertension, atrial fibrillation, male sex, and older age. This finding, therefore, re-emphasises the hitherto known fact that diabetes not only causes LVSD by being an important causative agent for ischaemic heart disease but also induces cardiac impairment by various other means leading to the so-called diabetic cardiomyopathy. ${ }^{21}$

Although a significant number of patients had hypertension, its association with the presence of LVSD among the patients in this cohort was not significant. In other studies, however, hypertension has been found to be an important predictor of cardiac impairment, particularly in patients with left ventricular hypertrophy. ${ }^{21} 22$ One possible explanation is that in our cohort, the majority of patients with hypertension were already taking appropriate treatment and were found to have reasonably controlled hypertension. It is therefore possible that the use of antihypertensive agents could have prevented or delayed the complications of hypertensive heart disease such as left ventricular hypertrophy or LVSD. However, we found that almost a fifth of the patients had echocardiographic evidence of hypertensive heart disease, which included the presence of left ventricular hypertrophy, abnormalities of diastolic filling, and various degrees of valvar abnormalities.

As previously shown, we found that a 12 lead ECG and a chest radiograph are useful tests for identifying patients with LVSD. ${ }^{23}{ }^{24}$ Hence, in our cohort, more patients with than without an abnormal ECG had left ventricular systolic impairment. Similarly, patients with cardiomegaly on chest radiography were more likely to have LVSD than were those with a normal or low cardiothoracic ratio. More significantly, however, the inference we derived from this work is the value of a normal ECG and a cardiothoracic ratio of less than 0.5 as tools for screening LVSD. Hence, although an abnormal ECG and increased cardiothoracic ratio are helpful in identifying patients with a high risk of heart failure, it is the absence of these abnormalities than can be used to screen a significant proportion of patients with preserved left ventricular systolic function in the community. Both of these tests have almost similar specificities and therefore can be used separately with relatively low negative predictive value or together, which significantly enhances their screening ability (table 5).

Some of our work and conclusions are unique when compared with similar work done previously. We showed for the first time the relation between the degree of LVSD and both ECG and chest radiograph (table 5). In previous studies, for example, a normal ECG was shown essentially to rule out LVSD. ${ }^{24}{ }^{25}$ In our work, we have clearly shown that a normal ECG can be used to rule out only substantial left ventricular systolic impairment (left ventricular ejection fraction less than $35 \%$ ), not borderline to moderate degrees of dysfunction (ejection fraction between 35-45\%). Similarly, a normal cardiothoracic ratio on a chest radiograph can screen out only significant LVSD. We have also shown that the combination of these two modalities, which are easily accessible to general

Table 5 Relation between left ventricular ejection fraction (LVEF) and the negative predictive values of normal ECG and CTR $<0.5$ separately and combined for predicting left ventricular dysfunction

\begin{tabular}{llll}
\hline LVEF & $\begin{array}{l}\text { Normal ECG (\%) } \\
(\mathbf{n}=586)\end{array}$ & $\begin{array}{l}\text { Normal chest radiograph } \\
(\mathbf{C T R}<\mathbf{0 . 5})(\%)(\mathbf{n}=\mathbf{4 7 2})\end{array}$ & $\begin{array}{l}\text { Normal ECG and chest radiograph } \\
(\%)(\mathbf{n}=\mathbf{3 4 0})\end{array}$ \\
\hline$<50 \%$ & 80.5 & 82.2 & 87.9 \\
$\leqslant 45 \%$ & 86.3 & 87.5 & 92.9 \\
$\leqslant 40 \%$ & 91.5 & 93.4 & 97.4 \\
$\leqslant 35 \%$ & 96.4 & 97.7 & 99.1 \\
\hline
\end{tabular}


Table 6 Proposed model for a hospital based heart failure service

Identification of high risk patients in the community

- Coronary heart disease registry (angina, previous myocardial infarction, coronary artery bypass graft)

- Patients with abnormal ECG particularly with $Q$ waves, left ventricular hypertrophy, and atrial fibrillation

- Patients with diabetes and hypertension

Screening

- 12 lead ECG

- Chest radiograph if available

- Possibly brain natriuretic peptide

Heart failure service

- Diagnostic service (technician led, five patients in a session, up to five sessions a week

- Heart failure treatment optimisation service (physician led, heart failure specialist nurse), 7-10 patients a session, two to three clinics a week)

Follow up

- General practitioners

- Possibly general practitioner cardiologists

- Heart failure specialist nurses

- Treatment optimisation clinic

practitioners, can further enhance their predictive value and therefore can be used together as a screening tool for heart failure in the community.

Another important aspect of this work is that, since it is a physician led clinic, it provided a diagnostic as well as a treatment optimisation service to community medical practitioners. In the later phase, a separate heart failure clinic was also set up, which is run by a middle grade physician and a heart failure specialist nurse. This has supplemented and improved the heart failure service. Hence, for a large number of patients, medications and overall management were optimised or altered (table 4). In the earlier phase (19941999), the emphasis was on adjusting the dose of diuretics and initiation of ACE inhibitor for patients with heart failure. Later, however, with the advent of newer heart failure treatment, $\beta$ blockers and spironolactone were also used more frequently by patients with stable heart failure. We did not stop ACE inhibitors in the majority of patients with normal left ventricular systolic function, as most of them had hypertension or significant valvar dysfunction. We also initiated anticoagulation for almost all patients with atrial fibrillation and in a few cases with significant LVSD (anterior wall hypokinesis, left ventricular thrombus).

We acknowledge certain shortcomings and weaknesses of our work. Firstly, this is a retrospective analysis of a service with no data on patients' morbidity and mortality. Important variables and factors such as patient's weight, body mass index, and smoking history have not been taken into account. The number of patients seen in one session was small and only 10-12 patients were seen in a typical month. The waiting list was unacceptably long at 4-6 months. There is also the issue of chest radiography. This test is expensive and not easily accessible.

We think, however, that with certain changes and modifications, this service can be adopted as a model for setting up similar clinics for the management of patients in the community. Such a service should be physician led but, to make it more cost effective and efficient, the diagnostic clinic should be run by an accredited echocardiographer. Hence, a relatively greater number of patients can be seen and their treatment optimised without a substantial waiting list. Patients' referral can be streamlined by the use of baseline investigations such as a 12 lead ECG and in some cases a chest radiograph and possibly determination of a natriuretic peptide such as brain natriuretic peptide. Potential patients with LVSD can be identified by screening patients' lists in the coronary heart disease registry, patients with the presence of one substantial (atrial fibrillation, left bundle branch block, or evidence of myocardial infarction on ECG) or at least two minor risk factors (hypertension, left ventricular hypertrophy, or other non-specific ECG abnormalities, diabetes), and patients with cardiac murmurs (table 6).

\section{Authors' affiliations}

S Shah, M K Davies, D Cartwright, Department of Cardiology, University Hospital Birmingham, Selly Oak Hospital, Birmingham, UK P Nightingale, Queen Elizabeth Medical Centre Edgbaston, Birmingham, UK

\section{REFERENCES}

1 Cowie MR, Wood DA, Coats AJW, et al. Incidence and aetiology of heart failure: a population-based study. Eur Heart J 1998;20:421-8

2 McMurray JJV, Hart W, Rhodes G. An evaluation of the cost of heart failure to the NHS in the UK. Br J Med Econ 1993;6:99-110.

3 Cowie MR, Mosterd A, Wood DA, et al. The epidemiology of heart failure. Eur Heart J 1997; 18:208-25.

4 Mosterd A, Hoes AW, de Bruyne MC, et al. Prevalence of heart failure and left ventricular dysfunction in the general population. Eur Heart $J$ 1999;20:447-55.

5 McDonagh TA, Morrison CE, Lawrence A, et al. Symptomatic and asymptomatic left-ventricular systolic dysfunction in an urban population. Lancet 1997;350:829-33.

6 Sans S, Kestesloot H, Kromhout D. The burden of cardiovascular diseases mortality in Europe. Task force of the European Society of Cardiology on cardiovascular mortality and morbidity statistics in Europe. Eur Heart $J$ 1997; 18:1231-48.

7 Cowie MR, Wood DA, Coats AJ, et al. Survival of patients with a new diagnosis of heart failure: a population based study. Heart 2000;83:505-10.

8 Bristow MR, Gilbert EM, Abraham WT, et al. Carvedilol produces doserelated improvements in left ventricular function and survival in subjects with chronic heart failure (MOCHA investigators). Circulation 1996:94:2807-16.

9 Marantz PR, Tobin JN, Wassertheil-Smoller S, et al. The relationship between left ventricular systolic function and congestive heart failure diagnosed by clinical criteria. Circulation 1988;77:607-12.

10 Hobbs FD, Jones Ml, Allan TF, et al. European survey of primary care physician perceptions on heart failure diagnosis and management (Euro-HF). Postgrad Med J 2001;77:765-8.

11 Wendelboe $\mathrm{O}$, Hansen JF, Hilden. The barriers to effective management of heart failure in general practice. J Eur Heart J 2000;21:1877-87.

12 McClure S, Caruana L, Davie AP, et al. Cohort study of plasma natriuretic peptides for identifying left ventricular systolic dysfunction in primary care. BMJ 1998:317:516-9.

13 Gupta SC. Congestive heart failure in the elderly: the therapeutic challenge of atypical presentations. Postgrad Med 1991;90:83-7.

14 Wheeldon NM, MacDonald TM, Flucker CJ, et al. Echocardiography in chronic heart failure in the community. Q J Med 1993;86:17-23.

15 Francis CM, Caruana L, Kearney $P$, et al. Open access echocardiography in management of heart failure in the community. BMJ 1995;310:634-6.

16 Colquhoun MC, Waine C, Monaghan MI, et al. Investigation in general practice of patients with suspected heart failure. Br Heart J 1995:74:335-6.

17 Ho KK, Pinsky JL, Kannel WB, et al. The epidemiology of heart failure: the Framingham Study. J Am Coll Cardiol 1993;22:6A-13A

18 McKee PA, Castelli WP, McNamara PM, et al. The natural history of congestive heart failure: the Framingham study. N Engl J Med $1971 ; 285: 1441-6$

19 Tepper D. Frontiers in congestive heart failure: tachycardia-related cardiomyopathy: a common cause of ventricular dysfunction in patients with atrial fibrillation referred for atrioventricular ablation. Congest Heart Fail 2000;6:284.

20 Kannel WB, Hjortland M, Castelli WP. Role of diabetes in congestive heart failure: the Framingham study. Am J Cardiol 1974;34:29-34.

21 Van Hoeven KH, Factor SM. A comparison of the pathological spectrum of hypertensive, diabetic, and hypertensive-diabetic heart disease. Circulation 1990;82:848-55

22 Kannel WB. Epidemiology and prevention of cardiac failure: Framingham study insights. Eur Heart J 1987; (suppl F):23-6.

23 Rihal CS, Davis KB, Kennedy JW, et al. The utility of clinical, electrocardiographic, and roentgenographic variables in the prediction of left ventricular function. Am J Cardiol 1995;75:220-3.

24 Davie AP, Francis CM, Love MP, et al. Value of the electrocardiogram in identifying heart failure due to left ventricular systolic dysfunction. BMJ 1996;312:222-5

25 Gillespie ND, McNeill G, Pringle T, et al. Cross sectional study of contribution of clinical assessment and simple cardiac investigations to diagnosis of left ventricular systolic dysfunction in patients admitted with acute dyspnoea. BMJ 1997;314:936-40. 